\title{
Analyzing the Preparation and Properties of Silver Nanoparticles; A Photo-Acoustic Study
}

\author{
F. M. Aldosari ${ }^{1}$, A. M. Azzeer ${ }^{2} \&$ A. M. Hassib ${ }^{2}$ \\ ${ }^{1}$ Physics Department, Applied and Natural Sciences, Prince Sattam Bin Abdulaziz University, Riyadh 11345, \\ Saudi Arabia \\ ${ }^{2}$ Physics and Astronomy Department, College of Science, King Saud University, Riyadh 11451, Saudi Arabia \\ Correspondence: F. M. Aldosari, Physics Department, Applied and Natural Sciences, Prince Sattam Bin \\ Abdulaziz University, Riyadh 11345, Saudi Arabia. E-mail: fah.aldosari@psau.edu.sa
}

Received: August 17, 2018

Accepted: October 20, 2018

Online Published: November 30, 2018

doi:10.5539/apr.v10n6p29

URL: https://doi.org/10.5539/apr.v10n6p29

\begin{abstract}
Metal nanoparticles have garnered recent attention due to their potential for use in various mechanical, electrical, chemical and optical applications. This study aimed to investigate the synthesis of silver nanoparticles using pulsed photo-acoustic (PA) spectroscopy techniques. The results indicated a linear relationship between absorbance and concentration. Additionally, stability of silver colloids was seen at room temperatures, with no aggregation. The nanoparticles were spherical and between 2-40 $\mathrm{nm}$ in diameter. Nanoparticle size and PA signal were inversely proportional. Furthermore, lack of nanoparticle stability was found to weaken the PA signal. Lastly, nanoparticle absorption was inversely proportional to fluorescence. Further studies are needed for exploring the rationale in the relationship between fluorescence and absorption of the nanoparticles.
\end{abstract}

Keywords: silver nanoparticles, photo-acoustic applications, fluorescence, size, stability

\section{Introduction}

Recent years have seen the use of metal nanoparticles in a wide variety of electrical, mechanical, optical, biological, and chemical applications (Stepanov, 2016). Metal nanoparticles have highly diverse uses due to their unique physical and chemical properties that occur due to the surface effect (Zhang et al., 2014). The optical properties of metallic nanoparticles are of great importance because of their ability to interact efficiently with optical fields over length scales smaller as compared to the diffraction limit and their sensitivity to changes within their local environment (Olson et al., 2015).

Among the noble-metal nanoparticles, silver nanoparticles have gained attention due to their varied uses as catalysts (Bindhu \& Umadevi, 2015) and photosensitive components (Dahiya, et al., 2015), in addition to their applications in surface-enhanced Raman spectroscopy (Grass et al., 2015). Furthermore, their property of optical absorption enables them to be used as highly competent contrast agents in photoacoustic imaging (Homan et al., 2010). Silver nanoparticles may be prepared in variety of ways; such as, chemical reduction by utilizing a reducing agent (Iravani, et al., 2014), electrochemical reduction (Nasretdinova et al., 2015), photochemical reduction (Iravani, et al., 2014) and finally thermal evaporation (Kibis et al., 2010), which is inclusive of chemical vapor deposition. Preparation may additionally be carried out physically by evaporating atoms from the surface of a metal by means of employing a high energy laser and then cooling to form nanoparticles (Abbasi et al., 2016).

A number of studies highlighted the effective role of the photoacoustic (PA) technique in determining the rate of nanoparticle production and concentration of those particles (Valverde-Alva et al., 2015). The use of this technique is highly recommended due to its ability to non-invasively identify concentrations of substances at high spatial resolutions (Taruttis \& Ntziachristos, 2015). Since nanoparticles are greatly size-dependent, there are dramatic changes in the electrical, optical, and magnetic properties because of reduced number of free electrons, smaller particle sizes, increased surface areas, and quantum confinement effect at the nano-scale (Zhou et al., 2017). Therefore, these properties need to be greatly investigated, so that silver nanoparticles may further be engineered at the appropriate sizes (Stepanov, 2016). As a result, silver nanoparticles with desired properties may be produced. However, there is a lack of studies that incorporate the use of this photoacoustic spectroscopy 
to identify the properties of silver nanoparticles. Therefore, this study has aimed to investigate the synthesis of silver nanoparticles using the laser-induced PA technique as a means to contribute to existing limited research in this context. Specifically, the concentration and size of the silver nanoparticles suspended in the dispersion were studied.

\subsection{Literature Review}

In previous literature, it was discussed that the PA technique effectively combined ultrasound and optical imaging modalities to study nanoparticle properties (Homan et al., 2010). The integration of PA with ultrasound modalities had been recommended by this study due to the complementary nature of these modalities to provide spatial images of high quality (Homan et al., 2010). The study found that the level of concentration of nanoparticles was in direct proportion to the intensity of the PA signal. Other studies have investigated the efficacy of this technique in studying the quantity and presence of nanoparticles within cells and tissues (Cook, Frey \& Emelianov, 2013). Additionally, the PA technique had been used to study the thermophysical characteristics of Al-doped zinc nanoparticles (El-Brolossy, Saber \& Ibrahim, 2013). This has earlier been corroborated by Homan et al. (2010), who suggested that PA was highly effective in detecting nanoparticles which were deep within tissue structures. Additionally, the use of pulsed PA has been noted in studying the optical properties of silver colloid nanoparticles through the use of laser ablation (Aldama-Reyna et al., 2018). The applicability of pulsed PA in studying the generation of silver nanoparticles in ethanol through the use of laser ablation had previously been highlighted (Valverde-Alva et al., 2015). This had not been seen in earlier studies, which had synthesized silver nanoparticles through the use of electrochemical means rather than PA (Nasretdinova et al., 2015). Additionally, the use of salt agents for synthesizing silver nanoparticles had previously been seen (Tolaymat et al., 2010). Moreover, previous studies had recruited the use of in situ reduction of hydroquinone in order to synthesize silver nanocomposities (Bao, Zhang \& Qi, 2011). Therefore, it was seen that the use of physical and chemical techniques was predominantly noted in order to effectively produce silver nanoparticles. This was corroborated by a more recent study, which highlighted that such techniques were inclusive of chemical vapor deposition, hydrothermal techniques, sol process, pyrolysis, chemical precipitation and micelle (Abbasi et al., 2016). The study had further discussed that the use of these techniques was significantly associated with complexities in the form of maintenance of stability of the nanoparticles and in achieving an appropriate size for the nanoparticles (Abbasi et al., 2016).

Silver nanoparticles were discussed to present comparatively better light absorptive properties as compared to gold nanoparticles; it therefore followed that the use of silver nanoparticles would be associated with comparatively stronger PA signals (Homan et al., 2010). Silver nanoparticles were additionally discussed to have therapeutic properties which were highly relevant to biomedical applications (Wei et al., 2015). Furthermore, the same study highlighted their applicability as anticancer and antiviral agents, and radio- and photo-sensitizers. Furthermore, they may be utilized for environmental and food safety applications due to their anti-microbial characteristics (Abbasi et al., 2016). The effectiveness of silver nanoparticle systems as competitive contrast agents in photoacoustic imaging had previously been highlighted (Homan et al., 2010). However, the use of silver nanoparticles may pose toxicity due to the increased level of silver being utilized, as highlighted by a toxicology study (Stensberg et al., 2011). Therefore, appropriate care is suggested when utilizing such nanoparticles. Further studies recommended the utilization of green strategies in order to synthesize silver nanoparticles (Andrade et al., 2016; Ahmed et al., 2016; Dhand et al., 2016).

\subsection{Hypotheses Development}

It may be clear that silver nanoparticle synthesis has gained immense attention due to their unique properties and potential for being utilized in a wide number of applications. However, there is still a gap in research pertaining to more explorations of silver nanoparticle synthesis through PA techniques. Thus, the following hypothesis has been developed.

$\mathbf{H}_{\mathbf{0}}$ : The properties of silver nanoparticles cannot be effectively determined using the PA technique.

$\mathbf{H}_{1}$ : The properties of silver nanoparticles can be determined effectively using the PA technique.

\section{Methods}

\subsection{Materials and Methods}

An ultrapure water purification system (Milli-Q Advantage A10, Millipore, USA) was used to prepare the aqueous in triple distilled water. Silver nitrate (AgNO3, 99\%, LA Container Inc., USA) and sodium borohydride (NaBH4, 97\%, Fluka, Switzerland) were used for this study. 


\subsection{Synthesis of Silver Nanoparticles}

To effectively reduce the ionic silver and stabilize the formed silver nanoparticles, a large excess of sodium borohydride is needed. Specifically, the initial concentration of sodium borohydride must be twice that of silver nitrate. When NaBH4 was varied from $2.0 \mathrm{mM}$, while using $1.0 \mathrm{mM}$ of AgNO3, the breakdown of the product took place in less than an hour. The weight of $\mathrm{AgNO} 3$ and $\mathrm{NaBH} 4$ in the solution were then found. Following this, the concentration of the silver nanoparticles in the solution was calculated.

To measure the sample masses, a balance (AL-204, D-SCALE INDONESIA) was used. Furthermore, Ultrasonic Equipment (Portable Cleaner, NXPC-1505, Ultrasonic frequency: 40Khz, Temp range: $0 \sim 70^{\circ} \mathrm{C}, \mathrm{KODO}$, Korea) was used to adequately mix the materials for approximately 5 minutes. In this way, a homogenous distribution of the particles is ensured. In order to cool down these materials, they were refrigerated through the use of an ice pan for approximately 10 minutes. In order to effectively homogenize the solution, a heating magnetic stirrer with timer (AREX.T, VELP, Italy) was utilized. Following this, a $10 \mathrm{~mL}$ volume of $1.0 \mathrm{mM}$ AgNO3 was added in a drop-wise manner at the rate of 1 drop/second to $30 \mathrm{~mL}$ of $2.0 \mathrm{mM} \mathrm{NaBH} 4$ solution. This solution had been cooled using an ice pan. A magnetic stir plate was used to carry out vigorous stirring of this mixture. Following the complete addition of AgNO3, the solution turned to a bright yellow color.

\subsection{PA Spectroscopy Technique}

For conducting this experiment, a Nd: YAG laser (model LQ 129, Solar Laser System) was used. This had a pump energy equal to $23 \mathrm{~J}$, a pulse energy of $280 \mathrm{~mJ}$ at $532 \mathrm{~nm}$, an output energy of $280 \mathrm{~mJ}$ at $532 \mathrm{~nm}$, a pulse repetition rate between $1-10 \mathrm{~Hz}$, a pulse width of $12 \mathrm{~ns}$ at $532 \mathrm{~nm}$, horizontal polarization at $532 \mathrm{~nm}$, delay of output pulse relative to the pump pulse equal to $154 \mu \mathrm{s}$, a beam divergence of $1 \mathrm{mrad}$ and a rod diameter of 8 $\mathrm{mm}$. A third harmonic generator (LG 103, Solar Laser System) was used with the laser. Furthermore, the association between the wavelength and the photoacoustic signal was evaluated through the use of a titanium-doped sapphire laser (LX 325, Solar Laser System). This had an output energy equivalent to $70 \mathrm{~mJ}$ at $755 \mathrm{~nm}$ and $40 \mathrm{~mJ}$ at $885 \mathrm{~nm}$. Furthermore, it had a tuning range between 694-935 nm and 866-1012 nm and a divergence equivalent to $1.5 \mathrm{mrad}$ at $755 \mathrm{~nm}$. Lastly, the linewidth was less than $0.8 \mathrm{~nm}$ at the maximum level of the tuning curveldequately obtain the UV-VIS absorption spectra of the nanoparticles, a UV-visible spectrophotometer (Lambda 40, Perkin Elmer, USA) was used. This obtained the spectra in the range of wavelengths between $190 \mathrm{~nm}$ to $1100 \mathrm{~nm}$. Furthermore, a halogen and deuterium lamp were used as sources of radiation, to encapsulate the spectrophotometer's range of the working wavelength. Additionally, five mirrors were used. The first, fourth and fifth mirrors were plane mirrors, whereas the second mirror was the toroidal mirror. Lastly, the third mirror was the spherical mirror. To procure the emission, excitation and synchronous fluorescence, a luminescence spectrometer (LS45, Perkin Elmer, USA) was utilized.

To increase the power of PA signal, a photodiode amplifier (PDA 6424, ILX Lightwave, USA) was used. Furthermore, a two-channel digital real-time oscilloscope (TDS 380, Tektronix Inc., USA) was used for observing the precise shape of the electrical signal received from the photodiode amplifier. To carry out spectral recording, an intensified charge coupled device (ICCD) camera (Andor iStar DH720-18F-03, Lot Oriel Instruments, USA) was utilized. This aids in achieving an enhanced response from 180-850 nm, 1024×256 pixels and $18 \mathrm{~mm}$ Gen 2 image intensifiers. Coupling of this ICCD camera was carried out with a monochromator (Oriel MS 257, model 77702, Lot Oriel Instruments, USA), a spectrograph with different gratings (300 and 1200 lines per $\mathrm{mm}$ ). To analyze the size of the nanoparticle distribution within the dispersion, a Microtrac S3500 tri-laser particle size analyzer (Microtrac S3500, Measuring Range 0.02 to 2800 Microns, Lasers Wavelength 780nm, Japan) was utilized. Furthermore, the nanoparticle shape, size and distribution were analyzed using a transmission electron microscopy (JEM-2100F field emission electron microscope, JEOL, Japan). The device is characterized by accelerating voltage between 80 to $200 \mathrm{kV}$ and a magnification level ranging from 50 to $1,500,000$. For analyzing and quantifying the phase, $x$-ray diffraction (X'Pert Pro, wavelength $1.54056 \AA$, PANalytical, Netherlands) was used. The diffraction technique was further used to ascertain the 3. r.setesuttructure of the sample using a high score.

This study has aimed to investigate the synthesis of silver nanoparticles in aqueous solution through the use of PA-induced laser excitation. The UV-Vis spectrum was attained with respect to a variety of sample concentrations. Additionally, various experimental parameters had been used in order to investigate the PA effect of silver nanoparticles. Figure 1 highlights the UV-Vis spectra of silver nanoparticles at various concentrations after 2880 minutes. 


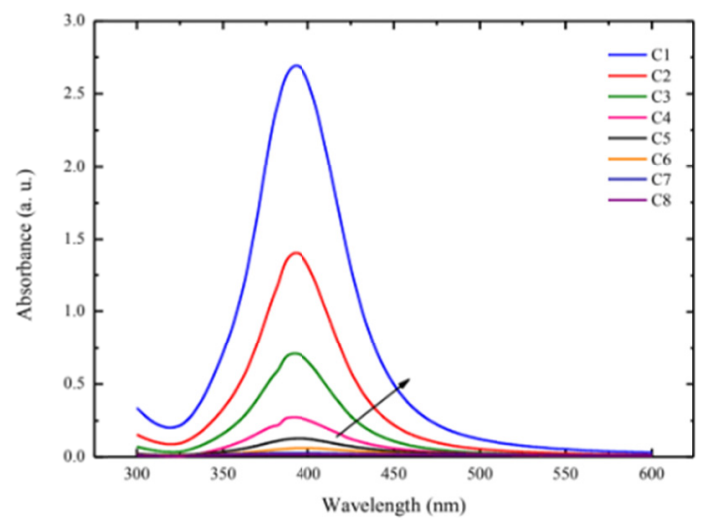

Figure 1. UV-Vis spectra of silver nanoparticles with different concentration after 2880 minutes

The results of the UV-Vis spectrum indicated that as the concentration of the silver nanoparticles in solution decreased, longer range wavelengths with respect to the absorption peaks were noted. The strongest absorption peak was detected at $392 \mathrm{~nm}$ with a concentration of $1.25 \times 10-3 \mathrm{M}$ (Figure 2). The relationship between the absorbance and concentration of silver nanoparticles with respect to stability in a time period between 1440 and 80640 minutes had been conducted. It was seen that there was a linear increase in absorbance as the concentrations increased.

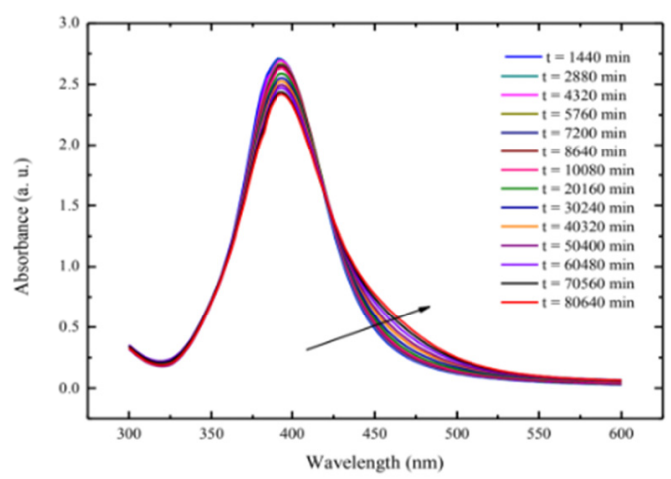

Figure 2. UV-Vis spectra of silver nanoparticles of concentration $1.25 \times 10-3 \mathrm{M}$ at different times

Furthermore, it was seen that the peak's position showed a slight red shift from $392 \mathrm{~nm}$ to $400 \mathrm{~nm}$ after four weeks, which indicated that larger particles with no aggregation were being formed. This absorbance of $400 \mathrm{~nm}$ was seen to be constant even following two months and no aggregation was noted. Therefore, this indicates towards the stability of silver colloids at room temperature conditions for several weeks or months. Concentrations of $3.9 \times 10-5 \mathrm{M}, 1.95 \times 10-5 \mathrm{M}$ and $9.76 \times 10-6 \mathrm{M}$ were however seen to behave differently; the results from TEM photographs indicated that the silver nanoparticles showed aggregation (Figure 3).
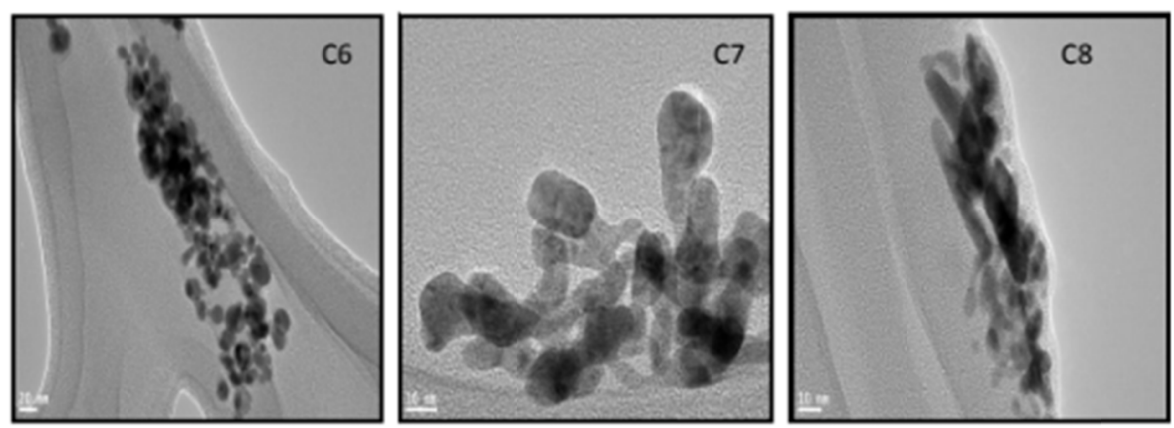

Figure 3. TEM photographs of aggregation of silver nanoparticles in concentrations $3.9 \times 10-5 \mathrm{M}, 1.95 \times 10-5$ $\mathrm{M}$ and $9.76 \times 10-6 \mathrm{M}$ 
The results of TEM further provided details pertaining to the size and morphology of silver nanoparticles, where it was seen that they are spherical in shape and approximately 2 to $40 \mathrm{~nm}$ in diameter (Figure 4).

$\mathrm{CU}$

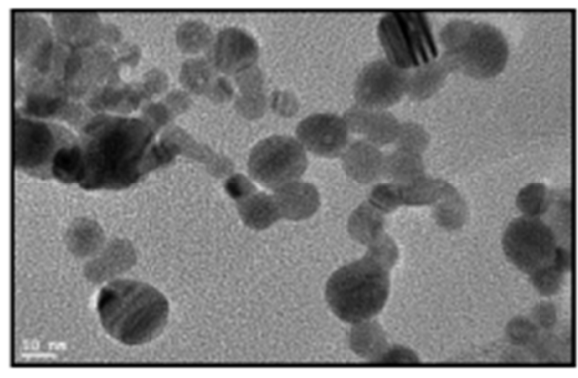

$\mathrm{C} 2$

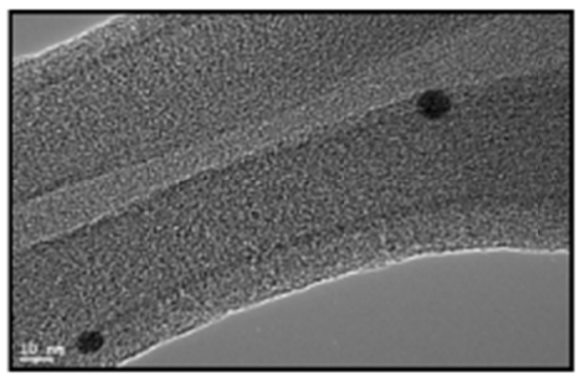

C4

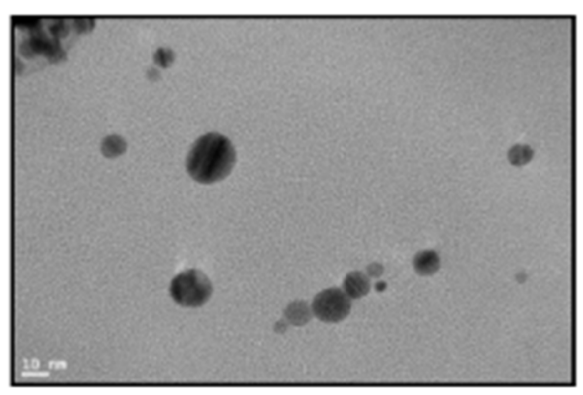

CI

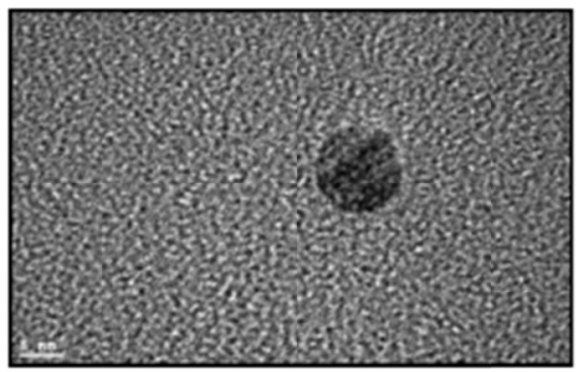

C3

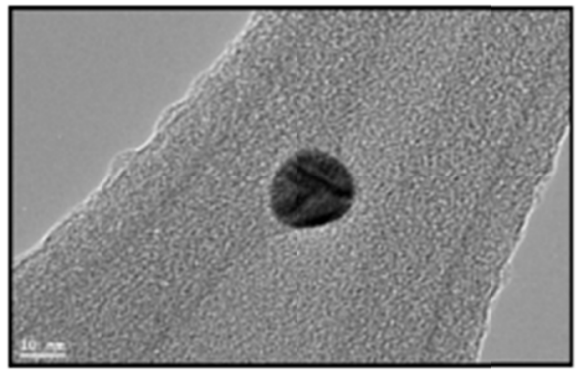

C5

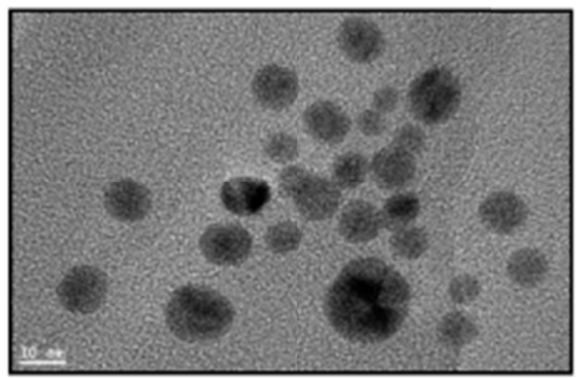

Figure 4. Typical TEM image of silver nanoparticles in water

The PA analysis indicated the results of the photoacoustic signal attained from these nanoparticles. As discussed earlier, a Ti-sapphire laser source was used for pumping the silver nanoparticles solution, where it was seen that increasing the PA signal was associated with increases in the concentration of silver nanoparticles in the solution. Specifically, the maximum peak for the PA signal was found at approximately $395 \mathrm{~nm}$ for each concentration except at $3.125 \times 10-4 \mathrm{M}$. Therefore, it was seen to be best to utilize the laser source for third harmonic generation having a wavelength of $395 \mathrm{~nm}$.

Furthermore, changes in the concentrations of the silver nanoparticles were investigated with respect to the PA signal. It was seen that increases in concentration were directly associated with increases in the PA signal until saturation. The greatest PA signal was noted with respect to a concentration of $1.25 \times 10-3 \mathrm{M}$ having a repetition rate of $2 \mathrm{~Hz}$ and at an energy of $30 \mathrm{~mJ}$. Moreover, the influence of nanoparticle size on the PA signal was investigated when excited using a laser of, $30 \mathrm{~mJ}$ energy, $355 \mathrm{~nm}$ wavelength and $2 \mathrm{~Hz}$ repetition. It was seen that decreasing the nanoparticle size was associated with increase in the PA signal (Figure 5). 


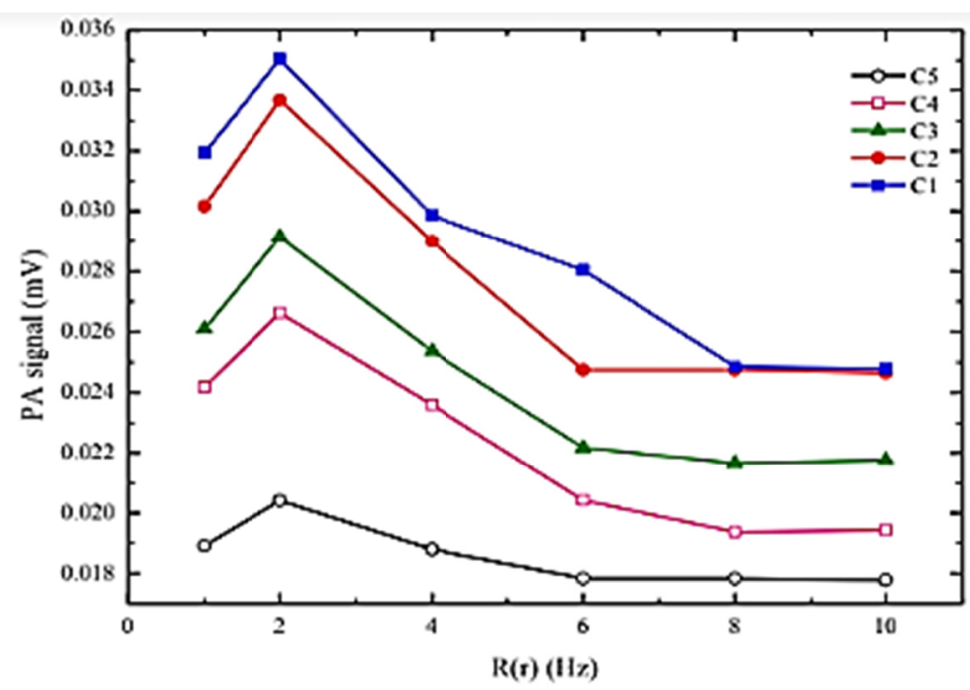

Figure 5. PA signal vs. $R(r)$ at $E=30 \mathrm{~mJ}$

The results from the fluorescence and absorbance of the PA signals indicated that the prepared silver nanoparticles lacked stability immediately following preparation, due to the presence of chemical reaction within the solution. This thereby resulted in inducing a weak PA signal. The following figures indicate the changes observed over time in the silver nanoparticle solution. Following two days, it was seen that a yellow color was noted for this solution, indicating particle suspension in the solution. Conversely, it was seen that silver nanoparticle absorption was greatest under the lowest levels of fluorescence. The PA signal was further seen to be strong, which was indicative of the suspension and stability of the particles in solution. The PA signal demonstrated stability for a duration of time, following which a decrease in silver nanoparticle absorption and increase in fluorescence was noted. Therefore, the PA signal decreases to the lowest value after approximately two months, which shows the aggregation of some nanoparticles at this time (Figure 6).

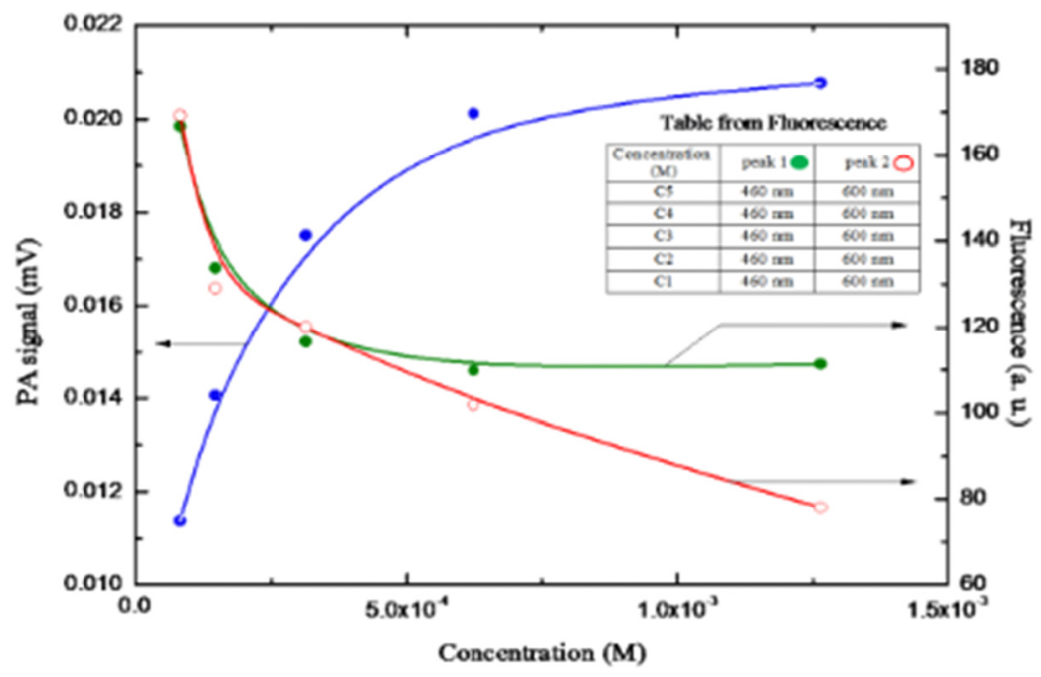

Figure 6. PA signal and fluorescence vs. concentration of silver nanoparticles for the two fluorescence peaks after 80640 minutes

\section{Discussion}

The use of PA techniques for investigating the characteristics of gold-silver nanoparticles had previously been highlighted (Hatef et al., 2015; Kim et al., 2018). Their use specific to silver nanoparticles had additionally been noted, albeit in a limited quantity of studies (Valverde-Alva et al., 2015). In the present study, there was a linear increase in absorbance as the concentrations of the nanoparticles in solution increased. The rationale behind this 
was discussed in an additional study which observed similar results (Kriel \& Priest, 2016). The study highlighted that according to Beer-Lambert's law, the absorbance of a solution is linearly proportional to the concentration. The results obtained by the present study are in accordance with this law.

Additionally, the slight red shift of the observed peaks over time was indicative of larger particles with no aggregation being formed; thereby, suggesting the stability of silver colloids at room temperature conditions. This was similarly highlighted in a study by Iravani et al. (2014), which explored the chemical, physical and biological means to synthesize silver nanoparticles. The lack of nanoparticle aggregation as seen in the present study is greatly advantageous, since it has been reported that the performance of nanoparticles is adversely impacted by increasing aggregation (Shi et al., 2015). However, the TEM results of the present study indicated that aggregation was present at higher nanoparticle concentrations, a finding which was consistent with that observed by Gharagozloo and Goodson (2010). The aforementioned study emphasized that increases in nanoparticle aggregation were significantly noted at higher concentrations and temperatures.

The results of the TEM were further elucidatory in that they indicated the size of the nanoparticles to be approximately 2 to $40 \mathrm{~nm}$ in diameter and their morphology as being spherical. The presence of spherical silver nanoparticles had earlier been observed in a study by Logeswari, Silambarasan and Abraham (2015), which had utilized chemical means to synthesize these particles. A further study highlighted that nanoparticles generally had sizes ranging between 10 to $20 \mathrm{~nm}$. It was further seen that an increase in the PA signal was directly correlated with increases in silver nanoparticle concentration until saturation, a finding which was consistent with an additional study by He et al. (2017). Furthermore, the present study found decreases in the size of nanoparticles to be significantly associated with increase in the PA signal, a finding which was in line with that observed by Hatef et al. (2015). Furthermore, the results from the absorbance and fluorescence of the PA signals illustrated a lack in stability of the silver nanoparticles immediately following preparation, due to which, a weak PA signal resulted. The close association between nanoparticle stability and increases in PA signals had earlier been explored in a study by Masim et al. (2016), which reported similar results. Conversely, it was also seen in the present study that the absorption of the silver nanoparticles in solution was maximized under the lowest levels of fluorescence. Further exploration is necessary for ascertaining the association between fluorescence and absorption of the silver nanoparticles.

To this end, this study filled the gap in existing research through suggesting a close association between variables such as absorbance and concentration, fluorescence and absorption, nanoparticle concentration and aggregation, PA signal and nanoparticle concentration and between nanoparticle size and PA signals. However, it was suggested that further studies are necessary for conducting a more comprehensive exploration of the relationship between fluorescence levels and the absorption of silver nanoparticles.

\section{Acknowledgements}

The author is very thankful to all the associated personnel in any reference that contributed in/for the purpose of this research. Further, this research holds no conflict of interest and is not funded through any source.

\section{References}

Abbasi, E., Milani, M., Fekri Aval, S., Kouhi, M., Akbarzadeh, A., Tayefi Nasrabadi, H., ... Samiei, M. (2016). Silver nanoparticles: synthesis methods, bio-applications and properties. Critical reviews in microbiology, 42, 173-180. https://doi.org/10.3109/1040841x.2014.912200

Ahmed, S., Ahmad, M., Swami, B. L., \& Ikram, S. (2016). Green synthesis of silver nanoparticles using Azadirachta indica aqueous leaf extract. Journal of Radiation Research and Applied Sciences, 9, 1-7. https://doi.org/10.1016/j.jrras.2015.06.006

Aldama-Reyna, W., Agreda-Delgado, J. F., Valverde-Alva, M. A., \& Angelats-Silva, L. M. (2018). Photoacoustic study of Changes in Optical Properties of Colloids with Silver Nanoparticles Produced by Laser Ablation. International Journal of Applied Engineering Research, 13, 1408-1414.

Andrade, F. A. C., de Oliveira Vercik, L. C., Monteiro, F. J., \& da Silva Rigo, E. C. (2016). Preparation, characterization and antibacterial properties of silver nanoparticles-hydroxyapatite composites by a simple and eco-friendly method. Ceramics international, 42, 2271-2280. https://doi.org/10.1016/j.ceramint. 2015.10.021

Bao, Q., Zhang, D., \& Qi, P. (2011). Synthesis and characterization of silver nanoparticle and graphene oxide nanosheet composites as a bactericidal agent for water disinfection. Journal of colloid and interface science, 360, 463-470. https://doi.org/10.1016/j.jcis.2011.05.009 
Bindhu, M. R., \& Umadevi, M. (2015). Antibacterial and catalytic activities of green synthesized silver nanoparticles. Spectrochimica Acta Part A: Molecular and Biomolecular Spectroscopy, 135, 373-378. https://doi.org/10.1016/j.saa.2014.07.045

Cook, J. R., Frey, W., \& Emelianov, S. (2013). Quantitative photoacoustic imaging of nanoparticles in cells and tissues. ACS nano, 7, 1272-1280. https://doi.org/10.1021/nn304739s

Dahiya, S., Bhardwaj, N., Bhardwaj, S., Mehta, J., Sehgal, A., \& Prasad, M. (2015). Silver nanoparticles embedded photosensitive silicon as catheter materials. Adv Anim Vet Sci, 3, 10-15.

Dhand, V., Soumya, L., Bharadwaj, S., Chakra, S., Bhatt, D., \& Sreedhar, B. (2016). Green synthesis of silver nanoparticles using Coffea arabica seed extract and its antibacterial activity. Materials Science and Engineering: C, 58, 36-43. https://doi.org/10.1016/j.msec.2015.08.018

El-Brolossy, T. A., Saber, O., \& Ibrahim, S. S. (2013). Determining the thermophysical properties of Al-doped $\mathrm{ZnO}$ nanoparticles by the photoacoustic technique. Chinese Physics B, 22, 074401. https://doi.org/10.1088/1674-1056/22/7/074401

Gharagozloo, P. E., \& Goodson, K. E. (2010). Aggregate fractal dimensions and thermal conduction in nanofluids. Journal of Applied Physics, 108, 074309. https://doi.org/10.1063/1.3481423

Grass, S., Diendorf, J., Gebauer, J. S., Epple, M., \& Treuel, L. (2015). Quantitative replacement of citrate by phosphane on silver nanoparticle surfaces monitored by surface-enhanced Raman spectroscopy (SERS). Journal of nanoscience and nanotechnology, 15, 1591-1596. https://doi.org/10.1166/jnn.2015.9143

Hatef, A., Darvish, B., Dagallier, A., Davletshin, Y. R., Johnston, W., Kumaradas, J. C., ... \& Meunier, M. (2015). Analysis of photoacoustic response from gold-silver alloy nanoparticles irradiated by short pulsed laser in water. The Journal of Physical Chemistry C, 119, 24075-24080. https://doi.org/10.1021/ acs.jpcc. 5 b08359

He, W., Ai, K., Jiang, C., Li, Y., Song, X., \& Lu, L. (2017). Plasmonic titanium nitride nanoparticles for in vivo photoacoustic tomography imaging and photothermal cancer therapy. Biomaterials, 132, 37-47. https://doi.org/10.1016/j.biomaterials.2017.04.007

Homan, K. A., Shah, J., Gomez, S., Gensler, H., Karpiouk, A. B., Brannon-Peppas, L., \& Emelianov, S. Y. (2010). Silver nanosystems for photoacoustic imaging and image-guided therapy. Journal of biomedical optics, 15, 021316.

Iravani, S., Korbekandi, H., Mirmohammadi, S. V., \& Zolfaghari, B. (2014). Synthesis of silver nanoparticles: chemical, physical and biological methods. Research in pharmaceutical sciences, 9, 385.

Kibis, L. S., Stadnichenko, A. I., Pajetnov, E. M., Koscheev, S. V., Zaykovskii, V. I., \& Boronin, A. I. (2010). The investigation of oxidized silver nanoparticles prepared by thermal evaporation and radio-frequency sputtering of metallic silver under oxygen. Applied Surface Science, 257, 404-413. https://doi.org/10.1016/j.apsusc.2010.07.002

Kim, T., Zhang, Q., Li, J., Zhang, L., \& Jokerst, J. V. (2018). A Gold/Silver Hybrid Nanoparticle for Treatment and Photoacoustic Imaging of Bacterial Infection. ACS nano. https://doi.org/10.1021/acsnano.8b01362

Kriel, F. H., \& Priest, C. (2016). Influence of sample volume and solvent evaporation on absorbance spectroscopy in a microfluidic "pillar-cuvette". Analytical Sciences, 32, 103-108. https://doi.org/10.2116/ analsci.32.103

Logeswari, P., Silambarasan, S., \& Abraham, J. (2015). Synthesis of silver nanoparticles using plants extract and analysis of their antimicrobial property. Journal of Saudi Chemical Society, 19, 311-317. https://doi.org/10.1016/j.jscs.2012.04.007

Masim, F. C. P., Liu, H. L., Porta, M., Yonezawa, T., Balčytis, A., Juodkazis, S., ... \& Hatanaka, K. (2016). Enhanced photoacoustics from gold nano-colloidal suspensions under femtosecond laser excitation. Optics Express, 24, 14781-14792. https://doi.org/10.1364/oe.24.014781

Nasretdinova, G. R., Fazleeva, R. R., Mukhitova, R. K., Nizameev, I. R., Kadirov, M. K., Ziganshina, A. Y., \& Yanilkin, V. V. (2015). Electrochemical synthesis of silver nanoparticles in solution. Electrochemistry Communications, 50, 69-72. https://doi.org/10.1016/j.elecom.2014.11.016

Olson, J., Dominguez-Medina, S., Hoggard, A., Wang, L. Y., Chang, W. S., \& Link, S. (2015). Optical characterization of single plasmonic nanoparticles. Chemical Society Reviews, 44, 40-57. https://doi.org/10. $1039 / \mathrm{c} 4 \operatorname{cs} 00131 \mathrm{a}$ 
Shi, Z., Tang, J., Chen, L., Yan, C., Tanvir, S., Anderson, W. A., ... \& Tam, K. C. (2015). Enhanced colloidal stability and antibacterial performance of silver nanoparticles/cellulose nanocrystal hybrids. Journal of Materials Chemistry B, 3, 603-611. https://doi.org/10.1039/c4tb01647e

Stensberg, M. C., Wei, Q., McLamore, E. S., Porterfield, D. M., Wei, A., \& Sepúlveda, M. S. (2011). Toxicological studies on silver nanoparticles: challenges and opportunities in assessment, monitoring and imaging. Nanomedicine, 6, 879-898. https://doi.org/10.2217/nnm.11.78

Stepanov, A. L. (2016). Nonlinear Optical Properties of Metal Nanoparticles in Silicate Glass. In Glass Nanocomposites (pp. 165-179). https://doi.org/10.1016/b978-0-323-39309-6.00007-9

Taruttis, A., \& Ntziachristos, V. (2015). Advances in real-time multispectral optoacoustic imaging and its applications. Nature Photonics, 9, 219. https://doi.org/10.1038/nphoton.2015.29

Tolaymat, T. M., El Badawy, A. M., Genaidy, A., Scheckel, K. G., Luxton, T. P., \& Suidan, M. (2010). An evidence-based environmental perspective of manufactured silver nanoparticle in syntheses and applications: a systematic review and critical appraisal of peer-reviewed scientific papers. Science of the Total Environment, 408, 999-1006. https://doi.org/10.1016/j.scitotenv.2009.11.003

Valverde-Alva, M. A., García-Fernández, T., Villagrán-Muniz, M., Sánchez-Aké, C., Castañeda-Guzmán, R., Esparza-Alegría, E., ... \& Herrera, C. M. (2015). Synthesis of silver nanoparticles by laser ablation in ethanol: a pulsed photoacoustic study. Applied Surface Science, 355, 341-349. https://doi.org/10.1016/j.apsusc.2015.07.133

Wei, L., Lu, J., Xu, H., Patel, A., Chen, Z. S., \& Chen, G. (2015). Silver nanoparticles: synthesis, properties, and therapeutic applications. Drug Discovery Today, 20, 595-601. https://doi.org/10.1016/j.drudis.2014.11.014

Zhang, W., Lu, G., Cui, C., Liu, Y., Li, S., Yan, W., ... \& Huo, F. (2014). A Family of Metal-Organic Frameworks Exhibiting Size-Selective Catalysis with Encapsulated Noble-Metal Nanoparticles. Advanced Materials, 26, 4056-4060. https://doi.org/10.1002/adma.201400620

Zhou, X., Jiang, Y., Chen, J., He, L., \& Zhang, L. (2017). Size-dependent nanoparticle dynamics in semiflexible ring polymer nanocomposites. Polymer, 131, 243-251. https://doi.org/10.1016/j.polymer.2017.10.038

\section{Copyrights}

Copyright for this article is retained by the author(s), with first publication rights granted to the journal.

This is an open-access article distributed under the terms and conditions of the Creative Commons Attribution license (http://creativecommons.org/licenses/by/4.0/). 